\title{
WELL-BOUNDED OPERATORS OF TYPE (B) IN A CLASS OF BANACH SPACES
}

\author{
WERNER RICKER
}

(Received 31 January 1986)

Communicated by R. O. Vyborny

\begin{abstract}
It is shown that in a Grothendieck space with the Dunford-Pettis property, the class of well-bounded operators of type (B) coincides with the class of scalar-type spectral operators with real spectrum. It turns out that in such Banach spaces, analogues of the classical theorems of Hille-Sz. Nagy and Stone concerned with the integral representation of $C_{0}$-semigroups of normal operators and strongly continuous unitary groups in Hilbert spaces, respectively, are of a very special nature.
\end{abstract}

1980 Mathematics subject classification (Amer. Math. Soc.): 47 B 40, 47 D 05.

Various notions of self-adjointness have been developed for operators acting in Banach spaces, each capturing in some respect the basic properties of self-adjointness in Hilbert space. One such notion is that of well-boundedness, a concept introduced by Smart, [17], and first studied by Smart and Ringrose, [15, 16, 17]; see [8] for a comprehensive treatment of such operators. Well-bounded operators are associated with certain monotone, projection-valued functions on the real line $\mathbf{R}$ (not necessarily given by a spectral measure) and have available a form of spectral diagonalization similar to, but in general weaker than, that for selfadjoint operators in Hilbert space, which make them suitable for treating conditional convergence. A slightly stronger notion available is that of well-bounded operator of type (B). Such operators have been intensively studied in various settings by Berkson, Gillespie and others; see [1], [3], [4], [5] and [9], for example.

Whereas there is a close analogy in certain respect between scalar-type spectral operators with real spectrum, in the sense of Dunford, and well-bounded operators of type (B), there are also fundamental differences, even in the Hilbert space

(C) 1987 Australian Mathematical Society $0263-6115 / 87 \$ A 2.00+0.00$ 
setting, [8, Chapter 18]. However, there is a class of (infinite dimensional) Banach spaces, namely, the Grothendieck spaces with the Dunford-Pettis property, briefly, GDP-spaces, in which scalar-type spectral operators are of a particularly simple form, [13]. Arguing by analogy, well-bounded operators of type (B) could also be expected to be of a simple form in such spaces. Indeed, this and more turns out to be the case: a continuous linear operator in a GDP-space is well-bounded of type (B) if and only if it is a scalar-type spectral operator with real spectrum (see Proposition 1). It follows that in GDP-spaces (see Corollary 1.1) the sum and product of commuting well-bounded operators of type (B) are again well-bounded of type (B); this is not the case in general, [8, Chapter 18]. Furthermore, in such spaces, a simple description of the classes of trigonometrically well-bounded operators and polar operators, introduced and studied in [1], is possible. In the final section it is shown that known analogues (see [1], for example) of the classical theorems of Hille-Sz. Nagy and Stone (concerned with the integral representation of $C_{0}$-semigroups of normal operators and strongly continuous unitary groups in Hilbert spaces, respectively) for the case of semigroups of well-bounded operators of type (B) and groups of trigonometrically well-bounded operators, in general Banach spaces, are of a very special nature in GDP-spaces.

\section{Preliminaries and notation}

If $X$ is a Banach space, then $L(X)$ denotes the space of all continuous linear operators of $X$ into itself. A spectral family in $X$, [1, Section 2], is a projectionvalued function $F: \mathbb{R} \rightarrow L(X)$ such that:

(i) $\sup \{\|F(\lambda)\|: \lambda \in \mathbb{R}\}<\infty$;

(ii) $F(\lambda) F(\mu)=F(\mu) F(\lambda)=F(\min \{\lambda, \mu\})$, for all $\lambda, \mu \in \mathbb{R}$;

(iii) $F$ is right-continuous on $\mathbf{R}$ in the strong operator topology;

(iv) $F$ has a left-hand limit in the strong operator topology at each point of $\mathbf{R}$;

(v) $F(\lambda) \rightarrow 0$ (resp. $F(\lambda) \rightarrow I$, the identity operator in $X$ ) in the strong operator topology as $\lambda \rightarrow-\infty$ (resp. $\lambda \rightarrow \infty$ ).

If there exist $a, b \in \mathbf{R}$ such that $F(\lambda)=0$ for $\lambda<a$ and $F(\lambda)=I$ for $\lambda \geqslant b$, then $F$ is said to be concentrated on $[a, b]$. An integration theory is available for spectral families, [8, Chapter 17]. In particular, if $J=[c, d]$ is a compact interval in $\mathbb{R}$ and $g$ is a continuous function of bounded variation on $J$, then $\int_{J} g d F$ exists as a strong limit of Riemann-Stieltjes sums. For such functions $g$, the operator $g(c) F(c)+\int_{J} g d F$ is denoted by $\int_{J}^{\oplus} g d F$.

Let $X$ be a Banach space. An element $T \in L(X)$ is said to be a well-bounded operator of type (B) if there exists a spectral family $F: \mathbb{R} \rightarrow L(X)$, necessarily 
unique, which is concentrated on some compact interval $[a, b]$ and

$$
T=\int_{[a, b]}^{\oplus} \lambda d F(\lambda),
$$

in which case $\sigma(T) \subseteq[a, b]$; see [1, Proposition 2.4], for example.

If $X$ is a Banach space, then an element $S$ of $L(X)$ is a scalar-type spectral operator, briefly, a scalar-operator, if $S=\int_{C} \lambda d P(\lambda)$ where $P: \mathscr{B} \rightarrow L(X)$ is a spectral measure, necessarily unique, defined on the Borel sets $\mathscr{B}$ of the complex plane $C$ and the integral is in the usual sense of integration with respect to a $\sigma$-additive vector measure, [11]. Of course, to say that $P$ is a spectral measure means that $P$ is $\sigma$-additive for the strong operator topology, $P(E \cap H)=$ $P(E) P(H)$ for every $E \in \mathscr{B}$ and $H \in \mathscr{B}$, and $P(C)=I$. A scalar-operator with real spectrum is necessarily well-bounded of type (B), [8, Theorem 16.17], but the converse is false in general, even if $X$ is a Hilbert space, [8, Chapter 18]. It may be interesting to note a partial converse: if $X$ is a Hilbert space and $T \in L(X)$ is a well-bounded operator of type (B) whose associated spectral family consists entirely of self-adjoint projections, then $T$ is necessarily a scalar-operator with real spectrum.

A Banach space $X$ is called a Grothendieck space if every sequence $\left\{x_{n}^{\prime}\right\}$ in the continuous dual space $X^{\prime}$, of $X$, which converges for the weak-star topology to zero converges weakly to zero. A Banach space $X$ is said to have the Dunford-Pettis property if $\lim _{n \rightarrow \infty}\left\langle x_{n}, x_{n}^{\prime}\right\rangle=0$ whenever $\left\{x_{n}\right\} \subseteq X$ tends weakly to zero and $\left\{x_{n}^{\prime}\right\} \subseteq X^{\prime}$ tends weakly to zero. Well known examples of GDP-spaces include $L^{\infty}$-spaces, $H^{\infty}(\mathbb{D})$, injective Banach spaces and certain $C(\Omega)$-spaces; see [12], for example. A complemented subspace of a GDP-space is again a GDP-space.

Let $X$ be a Banach space. A sequence of projections $\left\{P_{n}\right\}_{n=1}^{\infty}$ in $L(X)$ is said to be a Schauder decomposition if

(i) $P_{n} P_{m}=P_{\min (m, n)}$ for all integers $m, n \geqslant 1$,

(ii) $P_{n} \rightarrow I$ in the strong operator topology and

(iii) $P_{n} \neq P_{m}$ if $m \neq n$.

It is known that GDP-spaces do not admit a Schauder decomposition, [6], [12]. Accordingly, if $\left\{P_{n}\right\}$ is a sequence of projections in a GDP-space satisfying properties (i) and (ii) of the previous definition, then there exists a positive integer $N$ such that $P_{n}=I$ for every $n \geqslant N$.

LeMma 1 ([13]). Let $X$ be a GDP-space and $P: \mathscr{B} \rightarrow L(X)$ be a spectral measure. Then there exist finitely many commuting, pairwise disjoint projections $P_{1}, \ldots, P_{n}$ in the range of $P$, each one an atom, such that $I=\sum_{j=1}^{n} P_{j}$ and each operator $P(E), E \in \mathscr{B}$, is a partial sum of $\left\{P_{1}, \ldots, P_{n}\right\}$. In particular, $P$ assumes only finitely many values in $L(X)$. Accordingly, if $S \in L(X)$ is a scalar-operator with $P$ its resolution of the identity, then there exist complex numbers $z_{1}, \ldots, z_{n}$ such that $S=\sum_{j=1}^{n} z_{j} P_{j}$, where the $P_{j}, 1 \leqslant j \leqslant n$, are as above. 
Since spectral families are analogues of distribution functions of spectral measures supported on the real line, we might expect, in view of Lemma 1, that they also are of a very restricted type in GDP-spaces.

Lemma 2. Let $X$ be a $G D P$-space and $F: \mathbb{R} \rightarrow L(X)$ be a spectral family. Then $F$ is concentrated on a compact interval of $\mathbf{R}$ and its range $\{F(\lambda): \lambda \in \mathbf{R}\}$ is a finite subset of $L(X)$.

Proof. If $P_{n}=F(n)$, for each $n=1,2, \ldots$, then it follows from properties of $F$ that $\left\{P_{n}\right\}$ satisfies (i) and (ii) in the definition of Schauder decomposition and hence, there is $N$ such that $P_{n}=I$ for every $n \geqslant N$. By a similar argument, if $Q_{m}=I-F(-m)$, for each $m=1,2, \ldots$, then there is a positive integer $M$ such that $Q_{m}=I$ for every $m \geqslant M$. Using property (ii) of the definition of spectral family it follows that $F$ is concentrated in $[-M, N]$.

Suppose that the range of $F$ is infinite. Then there exists a monotonic sequence $\left\{\lambda_{n}\right\}$ in $[-M, N]$ such that $F\left(\lambda_{n}\right) \neq F\left(\lambda_{m}\right)$ whenever $n \neq m$. Suppose that $\left\{\lambda_{n}\right\}$ is strictly decreasing. Let $\lambda=\lim _{n \rightarrow \infty} \lambda_{n}$. By properties of $F$, the sequence of projections $R_{n}=I-F\left(\lambda_{n}\right), n=1,2, \ldots$, satisfies $R_{n} R_{m}=R_{\min (m, n)}$, for all $m, n \geqslant 1$. Furthermore, the right continuity of $F$ implies that $R_{n} \rightarrow(I-F(\lambda))$ in the strong operator topology. Now, the closed subspace $Y=(I-F(\lambda)) X$, of $X$, is invariant for each projection $R_{n}, n=1,2, \ldots$, and so its restriction $\tilde{R}_{n}$, to $Y$, is an element of $L(Y)$. Since $\tilde{R}_{n} \rightarrow \tilde{I}$ (the identity operator in $Y$ ) for the strong operator topology and $\tilde{R}_{n} \tilde{R}_{m}=\tilde{R}_{\min (m, n)}$, for all $m, n \geqslant 1$, with $Y$ being a GDP-space (as it is complemented in $X$ ), it follows that there exists an integer $K$ such that $\tilde{R}_{k}=\tilde{I}$ for every $k \geqslant K$, that is, $y=R_{k} y$ for every $y \in Y$ and $k \geqslant K$. Since elements of $Y$ are of the form $(I-F(\lambda)) x$, as $x$ varies through $X$, it follows, using the definition of the operators $\left\{R_{n}\right\}$, that $F\left(\lambda_{k}\right)=F(\lambda)$ for every $k \geqslant K$, which is a contradiction. The case when $\left\{\lambda_{n}\right\}$ is increasing can be argued similarly. Accordingly, $F$ has finite range.

\section{Well-bounded operators of type (B)}

Let $X$ be a GDP-space and $T \in L(X)$ be a well-bounded operator of type (B) with spectral family $F: \mathbb{R} \rightarrow L(X)$, concentrated on $[a, b]$, say. Then it follows from Lemma 2 and properties of $F$ that there exist finitely many points $\left\{\lambda_{1}, \ldots, \lambda_{m}\right\}$ with $a \leqslant \lambda_{1}<\lambda_{2}<\cdots<\lambda_{m} \leqslant b$ such that $F$ is constant in the interval $\left[\lambda_{j}, \lambda_{j+1}\right)$ and equal to $F\left(\lambda_{j}\right)$, for each $j \in\{1, \ldots, m\}, F$ is zero in $\left(-\infty, \lambda_{1}\right)$ and $F(\lambda)=I$ for $\lambda \in\left[\lambda_{m}, \infty\right)$. Accordingly, the spectrum $\sigma(T)$, of $T$, is contained in $\left\{\lambda_{j}\right\}_{j=1}^{m},[1$, Proposition 2.1(iii)], and so, in particular, $\sigma(T)$ is 
finite. It follows that $T$ is a spectral operator, [14, Proposition 2.2], and hence, $T$ is actually a scalar-operator, [8, Theorem 19.4]. In fact, it is clear from the definition of $\int_{[a, b]}^{\oplus} \lambda d F(\lambda)$ that

$$
T=\lambda_{1} F\left(\lambda_{1}\right)+\sum_{j=2}^{m} \lambda_{j}\left(F\left(\lambda_{j}\right)-F\left(\lambda_{j-1}\right)\right) .
$$

Accordingly, if $P: \mathscr{B} \rightarrow L(X)$ is defined by

$$
P(E)=\sum_{j=1}^{m} \delta_{j}(E) P_{j}, \quad E \in \mathscr{B},
$$

where $\delta_{j}$ denotes the Dirac point measure at $\lambda_{j}, 1 \leqslant j \leqslant m$, and $\left\{P_{j}\right\}_{j=1}^{m}$ are the commuting, pairwise disjoint projections defined by $P_{1}=F\left(\lambda_{1}\right)$ and $P_{j}=F\left(\lambda_{j}\right)$ $-F\left(\lambda_{j-1}\right)$, for each $2 \leqslant j \leqslant m$, then $P$ is a spectral measure and

$$
T=\int_{[a, b]} \lambda d P(\lambda)=\int_{\mathbf{R}} \lambda d P(\lambda)
$$

that is, $P$ is the resolution of the identity of $T$. Furthermore, $F(\lambda)=P((-\infty, \lambda])$, for each $\lambda \in \mathbb{R}$. These comments together with Theorems 16.17 and 17.15 of [8] establish the following

Proposition 1. Let $X$ be a GDP-space. Then an operator $T \in L(X)$ is well-bounded of type $(B)$ if and only if $T$ is a scalar-operator with real spectrum. In this case, $\sigma(T)$ is a finite subset of $\mathbf{R}$ and consists entirely of eigenvalues.

Combining Proposition 1 with [14, Proposition 2.1] gives the following

COROLLARY 1.1. The sum and product of commuting well-bounded operators of type (B) in a GDP-space are again well-bounded operators of type (B).

A continuous operator $T$ in a Banach space $X$ is said to be an AC-operator of type (B), [4, pages 316-318], if $T=C+i D$ where $C$ and $D$ are commuting well-bounded operators of type (B). In this case, $C$ and $D$ are unique, $[4$, Theorem 7].

Corollary 1.2. Let $X$ be a GDP-space. Then $T \in L(X)$ is an AC-operator of type (B) if and only if $T$ is a scalar-operator.

Proof. If $T$ is a scalar-operator, then $T=\operatorname{Re}(T)+i \operatorname{Im}(T)$ where $\operatorname{Re}(T)$ and $\operatorname{Im}(T)$ are commuting scalar-operators with real spectra and so Proposition 1 implies that $T$ is an AC-operator of type (B). The converse is clear from Proposition 1 and [14, Proposition 2.1]. 
Let $X$ be a Banach space. An operator $T$ in $L(X)$ is a polar operator, [1, Section 3], if there exist commuting, well-bounded operators $R$ and $A$, of type (B), such that $T=R \exp (i A)$. If $R=I$, then $T$ is called trigonometrically well-bounded, [1, page 433].

COROLlary 1.3. Let $X$ be a GDP-space and $T \in L(X)$. Then,

(i) $T$ is a polar operator if and only if $T$ is a scalar-operator, and

(ii) $T$ is trigonometrically well-bounded if and only if $T$ is a scalar-operator with $\sigma(T) \subseteq \mathbf{T}$, where $\mathbf{T}=\{z \in \mathbf{C} ;|z|=1\}$. In this case, there exist numbers $\lambda_{1}, \ldots, \lambda_{n}$ in $[0,2 \pi)$ and commuting, pairwise disjoint projections $P_{1}, \ldots, P_{n}$ in $L(X)$ such that $I=\sum_{j=1}^{n} P_{j}$ and

$$
T=\sum_{j=1}^{n} \exp \left(i \lambda_{j}\right) P_{j}
$$

Proof. follows easily from Remark (i) on page 436 of [1], Theorem 3.23(i) of [1], Proposition 2.1 of [14], and Lemma 1 and Proposition 1 above.

A continuous operator $T$ in a Banach space $X$ is called reflexive if the closed algebra generated by $I$ and $T$ with respect to the strong operator topology consists precisely of those operators in $L(X)$ which leave invariant each closed, $T$-invariant subspace of $X$. Scalar-operators are always reflexive, [10, Corollary 4.7]. C. Foias recently asked whether this is also the case for well-bounded operators of type (B); in general this is an open problem. However, it is immediate from the previous comment and Corollaries 1.2 and 1.3 that we do have the following

COROLlaRY 1.4. AC-operators of type (B) and polar operators in GDP-spaces are reflexive. In particular, well-bounded operators of type $(B)$ in GDP-spaces are reflexive.

\section{Classes of semigroups in GDP-spaces}

Certain generalizations of Sz. Nagy's theorem about semigroups of normal operators and Stone's theorem about groups of unitary operators in Hilbert space to the setting of semigroups and groups of scalar-operators in Banach spaces are known. Such extensions take on a particularly adequate form in the case of weakly sequentially complete Banach spaces (see [2, Theorem 4.2] and [18, Theorem 5.3], for example). There are also satisfactory analogues of these classical results for semigroups and groups consisting of well-bounded operators 
of type (B) and trigonometrically well-bounded operators, respectively; see Corollary 4.14 and Theorem 4.20 in [1]. In this section we examine these results in the particular setting of GDP-spaces where, of course, there is no distinction between well-bounded operators of type (B) and scalar-operators with real spectrum and between trigonometrically well-bounded operators and scalar-operators with spectrum in $\mathbf{T}$.

Let $X$ be a Banach space and $T:[0, \infty) \rightarrow L(X)$ be a $C_{0}$-semigroup consisting of scalar-operators with non-negative spectrum. Then there exists a spectral measure $P$, in $L(X)$, supported by an interval of the form $(-\infty, w]$ in $\mathbb{R}$ such that

$$
T(t)=\int_{-\infty}^{w} \exp (\lambda t) d P(\lambda), \quad t \geqslant 0,
$$

and, in particular, the infinitesimal generator $A$, of $T$, is a scalar-operator (usually unbounded) with spectrum in $(-\infty, w],[18$, Theorem 3.1]. In the case when $X$ is a GDP-space, it follows that $A$ is necessarily an element of $L(X)$; see [12, Theorem 6] or [14, Proposition 2.4], for example. Combining this observation with Lemma 1 establishes the following

Proposition 2. Let $X$ be a GDP-space and $T:[0, \infty) \rightarrow L(X)$ be a $C_{0}$-semigroup of scalar-operators, each with non-negative spectrum. Then there are real numbers $\lambda_{1}, \ldots, \lambda_{n}$ and commuting, pairwise disjoint projections $P_{1}, \ldots, P_{n}$ in $L(X)$ such that $I=\sum_{j=1}^{n} P_{j}$ and

$$
T(t)=\sum_{j=1}^{n} \exp \left(t \lambda_{j}\right) P_{j}, \quad t \geqslant 0 .
$$

If the hypothesis that each operator $T(t), t \geqslant 0$, have non-negative spectrum is relaxed, then it is still possible to conclude that

$$
T(t)=\int_{\mathbf{C}} \exp (t \lambda) d P(\lambda), \quad t \geqslant 0,
$$

for some spectral measure $P: \mathscr{B} \rightarrow L(X)$, not necessarily supported in $\mathbb{R}$ now, provided, for example, that the Banach space $X$ is weakly sequentially complete and the operators $T(t), t \geqslant 0$, have their resolutions of the identity uniformly bounded in norm, [18, Theorem 5.3]. Unfortunately, GDP-spaces do not fall into this scheme as they need not be weakly sequentially complete. Despite this, it is still the case that the same conclusion is valid (in an even stronger form).

Proposition 3. Let $X$ be a GDP-space and $T:[0, \infty) \rightarrow L(X)$ be a $C_{0}$-semigroup of scalar-operators. Then there exist complex numbers $\lambda_{1}, \ldots, \lambda_{n}$ and commuting, pairwise disjoint projections $P_{1}, \ldots, P_{n}$ in $L(X)$ such that $I=\sum_{j=1}^{n} P_{j}$ and

$$
T(t)=\sum_{j=1}^{n} \exp \left(t \lambda_{j}\right) P_{j}, \quad t \geqslant 0
$$


Proof. By Theorem 6 of [12] and Lemma 5.2 of [18], it follows that the generator of $T(\cdot)$ is a scalar-operator and belongs to $L(X)$. The conclusion then follows from [18, Theorem 2.1] and Lemma 1.

Remark. It is not assumed in Proposition 3 that the resolutions of the identity of the operators $T(t), t \geqslant 0$, are uniformly bounded in norm. Indeed, this follows as a consequence since, for each $t \geqslant 0$, the resolution of the identity of $T(t)$ is contained in the resolution of the identity of the generator for the semigroup.

The next result is an analogue of the well-known Stone theorem, formulated in [2], for example, in the setting of weakly sequentially complete spaces.

Proposition 4. Let $X$ be a GDP-space and $T: \mathbf{R} \rightarrow L(X)$ be a strongly continuous group of scalar-operators, each with spectrum contained in $\mathrm{T}$. Then the infinitesimal generator $A$, of $T$, belongs to $L(X)$, is a scalar-operator with (finite) spectrum in $i \mathbb{R}$ and

$$
T(t)=\int_{\mathbf{R}} \exp (i t \lambda) d P(\lambda)=\sum_{j=1}^{n} \exp \left(i t \lambda_{j}\right) P\left(\left\{\lambda_{j}\right\}\right), \quad t \in \mathbf{R},
$$

where $P: \mathscr{B}(\mathbf{R}) \rightarrow L(X)$ is the resolution of the identity of -iA (supported by the subset $\left\{\lambda_{j}\right\}_{j=1}^{n}$ of $\left.\mathbf{R}\right)$ and $\mathscr{B}(\mathbf{R})$ denotes the Borel subsets of $\mathbf{R}$.

Proof. Arguing as in the proof of Proposition 3 it is easily established that

$$
T(t)=\int_{\mathbf{C}} \exp (\lambda t) d Q(\lambda), \quad t \in \mathbb{R},
$$

where $Q: \mathscr{B} \rightarrow L(X)$ is the resolution of the identity of $A$, necessarily a scalar-operator in $L(X)$. Lemma 1 implies that $\sigma(A)$ is a finite subset of $\mathbb{C}$, say $\left\{z_{1}, \ldots, z_{n}\right\}$. Since each operator $T(t), t \in \mathbf{R}$, has spectrum in $\mathbf{T}$ it follows from the spectral mapping theorem and (1) that

$$
\sigma(T(t))=\left\{\exp \left(t z_{j}\right): 1 \leqslant j \leqslant n\right\} \subseteq \mathbb{T}, \quad t \in \mathbb{R},
$$

from which it is clear that $\left\{z_{j}\right\}_{j-1}^{n} \subseteq i \mathbb{R}$. The conclusion then follows from (1), now known to be an integral over $i \mathbf{R}$ (actually, over $\sigma(A)$ ), by making the change of variable $\lambda=i \xi, \xi \in \mathbb{R}$.

We conclude with a simple result which is not related to scalar-operators, but is, nevertheless, in the spirit of this section and is, perhaps, of independent interest. An important problem in the theory of $C_{0}$-semigroups in Banach spaces is to decide whether

$$
s(A, T)=\omega_{0}(T)
$$


for the generator $A$, of $T$, where $s(A, T)=\sup \{\operatorname{Re}(\lambda): \lambda \in \sigma(A)\}$ is the spectral bound of $A$ and $\omega_{0}(T)=\inf \left\{t^{-1} \ln \|T(t)\|: t>0\right\}$ is the growth bound of $T$. Semigroups satisfying (2) are called stable. Whereas it is always the case that $s(A, T) \leqslant \omega_{0}(T),[7$, Section 1], it is known that (2) is not valid in general, even for semigroups in Hilbert space, [20].

Proposition 5. Every $C_{0}$-semigroup in a GDP-space is stable.

Proof. By Theorem 6 of [12] the generator of the semigroup is a bounded operator. The result then follows from [19, page 387, Comment (i)].

The support of a Queen Elizabeth II Fellowship is gratefully acknowledged.

\section{References}

[1] H. Benzinger, E. Berkson and T. A. Gillespie, 'Spectral families of projections, semigroups, and differential operators', Trans. Amer. Math. Soc. 275 (1983), 431-475.

[2] E. Berkson, 'Semigroups of scalar-type operators and a theorem of Stone', Illinois J. Math. 10 (1966), 345-352.

[3] E. Berkson, 'Spectral families of projections in Hardy spaces', J. Funct. Anal. 60 (1985), 146-167.

[4] E. Berkson and T. A. Gillespie, 'Absolutely continuous functions of two variables and well-bounded operators', J. London Math. Soc. (2) 30 (1984), 305-321.

[5] E. Berkson and T. A. Gillespie, 'AC functions on the circle and spectral families', J. Operator Theory 13 (1985), 33-47.

[6] D. D. Dean, 'Schauder decompositions in (m)', Proc. Amer. Math. Soc. 18 (1967), 619-623.

[7] R. Derndinger, 'Über das Spectrum positiver Generatoren', Math. Z. 172 (1980), 281-293.

[8] H. R. Dowson, Spectral theory of linear operators (London Math. Soc. Monograph No. 12, Academic Press, London, 1978).

[9] T. A. Gillespie, 'A spectral theorem for $L^{p}$ translations', J. London Math. Soc. (2) 11 (1975), 499-508.

[10] T. A. Gillespie, 'Boolean algebras of projections and reflexive algebras of operators', Proc. London Math. Soc. (3) 37 (1978), 56-74.

[11] I. Kluvanek and G. Knowles, Vector measures and control systems (North-Holland, Amsterdam, 1976).

[12] H. P. Lotz, 'Tauberian theorems for operators on $L^{\infty}$ and similar spaces, Functional analysis: Surveys and recent results III, pp. 117-133, (North-Holland Series No. 90, Amsterdam, 1984).

[13] W. Ricker, 'Spectral operators of scalar-type in Grothendieck spaces with the Dunford-Pettis property', Bull. London Math. Soc. 17 (1985), 268-270.

[14] W. Ricker, 'Spectral operators and weakly compact homomorphisms in a class of Banach spaces', Glasgow Math. J. 28 (1986), 215-222.

[15] J. R. Ringrose, 'On well-bounded operators', J. Austral. Math. Soc. 1 (1960), 334-343.

[16] J. R. Ringrose, 'On well-bounded operators II,' Proc. London Math. Soc. (3) 13 (1963), 613-638.

[17] D. R. Smart, 'Conditionally convergent spectral expansions', J. Austral. Math. Soc. 1 (1960), 319-333. 
[18] A. R. Sourour, 'Semigroups of scalar-type operators on Banach spaces', Trans. Amer. Math. Soc. 200 (1974), 207-232.

[19] R. Triggiani, 'On the stabilizability problem in Banach spaces', J. Math. Anal. Appl. 52 (1975), 383-403.

[20] J. Zabczyk, 'A note on $C_{0}$-semigroups', Bull. Acad. Polon. Sci. 23 (1975), 895-898.

Centre for Mathematical Analysis

Australian National University

Canberra, 2600

Australia 